\title{
Electronic structure of noncrystalline transition metal silicate and aluminate alloys
}

\author{
G. Lucovsky, ${ }^{\text {a) }}$ G. B. Rayner, Jr., D. Kang, G. Appel, R. S. Johnson, Y. Zhang, \\ D. E. Sayers, and H. Ade \\ Department of Physics, North Carolina State University, Raleigh, North Carolina 27695-8202
}

J. L. Whitten

Department of Chemistry, North Carolina State University, Raleigh, North Carolina 27695-8202

(Received 1 May 2001; accepted for publication 30 July 2001)

\begin{abstract}
A localized molecular orbital description (LMO) for the electronic states of transition metal (TM) noncrystalline silicate and aluminate alloys establishes that the lowest conduction band states are derived from $d$ states of TM atoms. The relative energies of these states are in agreement with the $\mathrm{LMO}$ approach, and have been measured by $\mathrm{x}$-ray absorption spectroscopy for $\mathrm{ZrO}_{2}-\mathrm{SiO}_{2}$ alloys, and deduced from an interpretation of capacitance-voltage and current-voltage data for capacitors with $\mathrm{Al}_{2} \mathrm{O}_{3}-\mathrm{Ta}_{2} \mathrm{O}_{5}$ alloy dielectrics. The LMO model yields a scaling relationship for band offset energies providing a guideline for selection of gate dielectrics for advanced Si devices. (C) 2001 American Institute of Physics. [DOI: 10.1063/1.1404997]
\end{abstract}

Noncrystalline alloys of group IIIB, IVB, and VB transition metal (TM) oxides with $\mathrm{SiO}_{2}$ and $\mathrm{Al}_{2} \mathrm{O}_{3}$, have been proposed as alternative gate dielectrics for advanced $\mathrm{Si}$ devices. ${ }^{1}$ Interest in these alloys derives from their high dielectric constants, $k$, relative to $\mathrm{SiO}_{2}$, as well as their good thermal stability. Higher values of $k$ allow use of physically thicker films to obtain the same effective capacitance as in $\mathrm{SiO}_{2}$ devices, and thus provides potential for significantly reduced direct tunneling. However, decreases in tunneling are mitigated in part by lower conduction band offset energies that define the barrier between the $c$-Si substrate and the dielectric. ${ }^{2}$ This letter identifies a relationship between conduction band offset energies with respect to $c$-Si, ${ }^{2,3}$ and the energy difference between the TM atomic $n+1 s$ and $n d$ states providing a useful guideline for selection of alternative gate dielectrics.

The calculations in Ref. 2 demonstrated that the lowest conduction bands in crystalline TM oxides are derived from atomic $d$ states of TM atoms. The experimental results of Ref. 3 and this letter, and the localized molecular orbital (LMO) model results presented below show that this assignment is not restricted to crystalline oxides, but applies more generally to noncrystalline TM oxides as well. In the LMO model, orbital energies of the lowest antibonding TM $d$ states are determined predominantly by the coordination and symmetry of the TM atom, and are at energies approximately equal to the normalized TM atom $d$ states. ${ }^{4,5}$

Figure 1 displays $\mathrm{x}$-ray absorption spectra (XAS) for a series of $\mathrm{Zr}$ silicate alloys, $\left(\mathrm{ZrO}_{2}\right)_{x}\left(\mathrm{SiO}_{2}\right)_{1-x}$, as obtained at beam line U4B at the National Synchrotron Light Source. Based on dipole selection rules, spectral features are assigned to transitions between $M_{2,3} p$-core states of the $\mathrm{Zr}$ atoms, and conduction band states derived from $4 d$ ( $\mathrm{a}$ and $\mathrm{b}$, and $\mathrm{a}^{\prime}$ and $\mathrm{b}^{\prime}$ ) and $5 s$ ( $\mathrm{c}$ and $\left.\mathrm{c}^{\prime}\right) \mathrm{Zr}$ atomic states. The local bonding of $\mathrm{Zr}$ and $\mathrm{Si}$ atoms in these alloys has been ad-

a)Electronic mail: gerry_lucovsky@ncsu.edu dressed in Refs. 6 and 7. Plasma-deposited films prepared at $300{ }^{\circ} \mathrm{C}$ are noncrystalline alloys with $\mathrm{Si}-\mathrm{O}$ and $\mathrm{Zr}-\mathrm{O}$ bonds, but no detectable $\mathrm{Zr}-\mathrm{Si}$ bonds. ${ }^{8}$ After annealing at $1000^{\circ} \mathrm{C}$, the $x=0.5$ alloy separates into amorphous $\mathrm{SiO}_{2}$ and crystalline $\mathrm{ZrO}_{2}$, and the $x=1.0$ end member, $\mathrm{ZrO}_{2}$, also crystallizes. The energies of features highlighted in the XAS spectra of Fig. 1 are independent of alloy composition and crystallinity to within an experimental uncertainty of $\pm 0.1 \mathrm{eV}$.

Figure 2 is a schematic energy level diagram for a group IV TM atom bonded octahedrally to six $\mathrm{O}$ atoms. The top of the valence band is derived from nonbonding $\pi$ orbitals of $\mathrm{O}$ $2 p$ states. The lowest conduction bands are associated with TM $n d$ states with $t_{2 g}\left(\pi^{*}\right)$ and $e_{g}\left(\sigma^{*}\right)$ symmetries. The next conduction band with $a_{1 g}\left(\sigma^{*}\right)$ symmetry is derived from TM $n+1 s$ states. Three distinct conduction bands have been previously reported in XAS studies of crystalline $\mathrm{TiO}_{2},{ }^{8}$ and $\mathrm{TiS}_{2}$ (Ref. 9) in which the $\mathrm{Ti}$ atoms are octahedrally

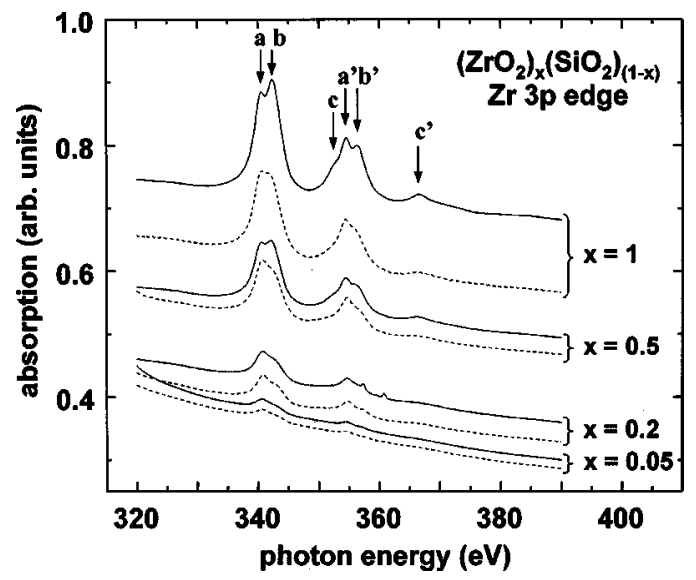

FIG. 1. X-ray absorption spectra for excitation from $\mathrm{ZrM}_{2,3} p$ states into empty $\mathrm{Zr} 4 d$ and $5 s$ states in $\mathrm{Zr}$ silicate alloys before (dashed) and after a rapid thermal anneal at $1000^{\circ} \mathrm{C}$ (solid). a, b, and $\mathrm{c}$ and $\mathrm{a}^{\prime}, \mathrm{b}^{\prime}$, and $\mathrm{c}^{\prime}$ designate energy differences between $M_{2}$ and $M_{3} p$ states, respectively, and the antibonding $\mathrm{Zr}$ states. Two sharp features in the $x=0.2$ plot are assigned to Ca contamination of unknown origin. 


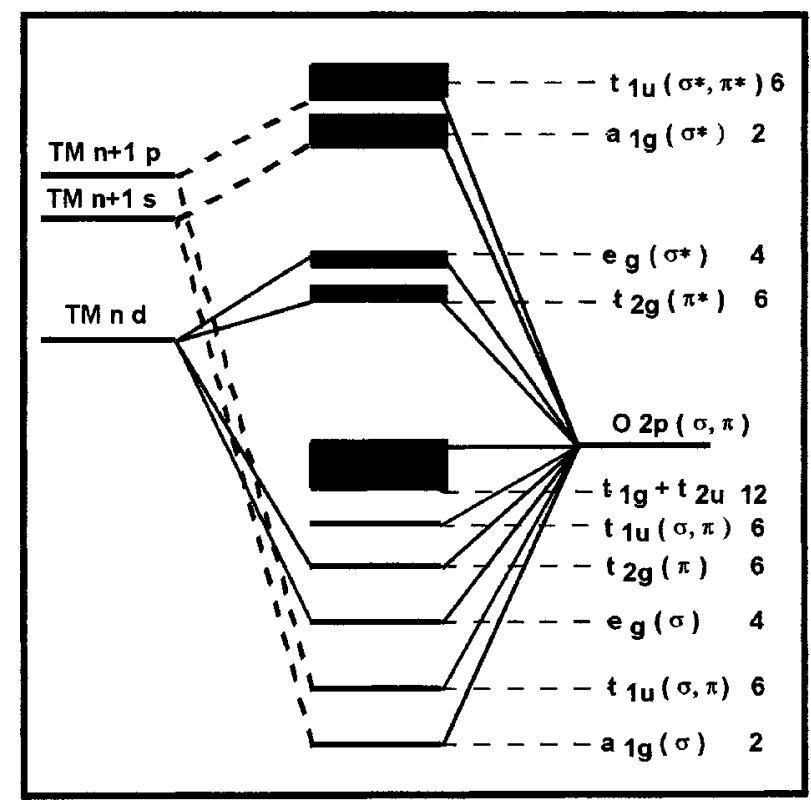

FIG. 2. Relative orbital energies for a group IVB transition metal, TM, in an octahedral bonding geometry with $\mathrm{O}$ atom neighbors (Refs. 5-7). The integers appearing after the symmetry designations are the total number of electrons accommodated in each orbital.

coordinated. These spectra demonstrate (i) narrow widths for the lowest conduction bands associated with TM $d$ states, and (ii) an energy gap between the $3 d$ - and $4 s$-derived bands. Similar features are also evident in Fig. 1 in both crystalline and noncrystalline films. The symmetry designations of the first two antibonding bands labeled $\mathrm{a}$ and $\mathrm{b}$, and $\mathrm{a}^{\prime}$ and $\mathrm{b}^{\prime}$ in Fig. 1, with $e\left(\pi^{*}\right)$ and $T_{2}\left(\sigma^{*}\right)$ symmetry, respectively, are reversed with respect to $\mathrm{TiO}(\mathrm{S})_{2}$, due to differences in TM coordination, ${ }^{4,6}$ four at low $\mathrm{Zr}$ concentrations and increasing to eight at higher concentrations, ${ }^{7}$ as compared to six in the Ti materials. ${ }^{8,9}$ The next conduction band, $\mathrm{c}$ and $\mathrm{c}^{\prime}$ in Fig. 1, is derived from TM $s$ states, and is independent of coordination.

In the LMO model of Fig. 2, the top of the valence band is at the orbital energy of an $\mathrm{O} 2 p$ state. The lowest conduction band state is associated with an antibonding TM $\pi^{*} d$ state and is at an energy approximately equal to the TM atomic $d$-state energy. The energy difference between the lowest conduction edge, $t_{2 g}\left(\pi^{*}\right)$, and the bottom of the $a_{1 g}\left(\sigma^{*}\right)$ band scales with the difference in energy between the TM atom $n+1 s$ and $n d$ states. Similar LMO energy level schemes apply to octahedrally coordinated TM dichalcogenides in which $\mathrm{S}$ and Se replace O. ${ }^{10,11}$

Figure 3(a) displays optical band gap versus atomic $p$-state energy of $\mathrm{O}, \mathrm{S}$, and Se, respectively, for $\mathrm{HfO}_{2}, \mathrm{HfS}_{2}$, and $\mathrm{HfSe}_{2},{ }^{2,10}$ and $\mathrm{MnO}, \mathrm{MnS}$, and MnSe. ${ }^{11}$ The linear scaling is consistent with the LMO model. ${ }^{12}$ Scaling is extended in Fig. 3(b) to band offset energies, where comparisons are made between the LMO theory and reported results. ${ }^{2,3}$ The band conduction band offset energy between Si and a high- $k$ dielectric defines the barrier for direct tunneling, and/or thermal emission from an $n^{+} \mathrm{Si}$ substrate into the dielectric. In a TM alloy such as $\mathrm{Al}_{2} \mathrm{O}_{3}-\mathrm{Ta}_{2} \mathrm{O}_{5}$, or $\mathrm{SiO}_{2}-\mathrm{ZrO}_{2}$, the offset also defines the relative energy of possible TM trapping states. ${ }^{13}$ Since the energies of TM metal $n+1 s$ states are at about the same energy, approximately $-5.5 \pm 0.2 \mathrm{eV}$, for

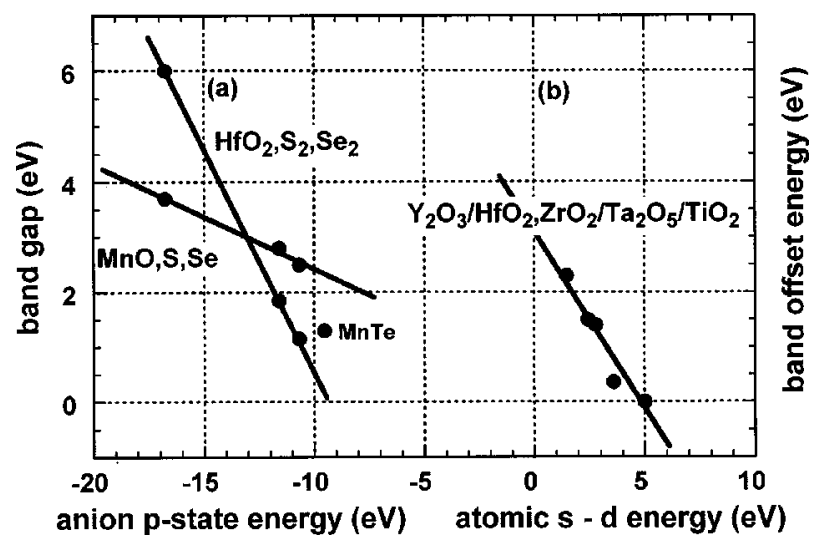

FIG. 3. (a) Optical band gap vs energy of atomic $p$ states of $\mathrm{O}, \mathrm{S}, \mathrm{Se}$ for (i) $\mathrm{HfO}_{2}, \mathrm{HfS}_{2}$, and $\mathrm{HfSe}_{2}$ (Ref. 9), and (ii) $\mathrm{MnO}, \mathrm{MnS}$, and MnSe (Ref. 10). The MnTe point falls below the linear fit because of overlap between Mn $d$ and Te $p$ states (Ref. 10). (b) Band offset energies (Refs. 2 and 3) vs the absolute value of the $n+1 s$ and $n d$ energy difference for TM elemental oxides and silicates.

TMs being considered for gate dielectrics, e.g., Hf, Zr, and Y, and since $n+1 s-n d$ state energy differences are in excess of $1.5 \mathrm{eV}$, this anticipates an additional scaling relationship between the conduction band edges of $c$-Si and TM oxides and alloys as shown in Fig. 3(b).

Interpretation of electrical data for capacitors with $\mathrm{Al}_{2} \mathrm{O}-\mathrm{Ta}_{2} \mathrm{O}_{5}$ dielectrics also shows that relative energies of Ta antibonding $d$ states are not significantly changed by alloying. ${ }^{13}$ For example, the plot of leakage current versus 1/temperature in Fig. 4 indicates two distinct activation energies. In the context of Fig. 2, and as discussed in Ref. 13, the activation energy of $\sim 0.3 \mathrm{eV}$ in the low temperature regime is for electron injection into interfacial traps associated with antibonding Ta $d$ states. The $\sim 1.5 \mathrm{eV}$ activation energy at higher temperatures is associated with emission out of these localized Ta traps into extended conduction band states with $\mathrm{Al} 3 s\left(\sigma^{*}\right)$ character as in a Poole-Frenkel transport process. The activation energies are consistent with x-ray photoelectron spectroscopy (XPS) determinations of band offset energies for noncrystalline $\mathrm{Ta}_{2} \mathrm{O}_{5}$ and $\mathrm{Al}_{2} \mathrm{O}_{3}{ }^{3}$

This letter has presented experimental data in Fig. 1 for the energies of antibonding transition metal $d$-states $\mathrm{ZrO}_{2}-\mathrm{SiO}_{2}$ binary alloys. The independence of energies of spectral features on alloy composition, and crystallinity are

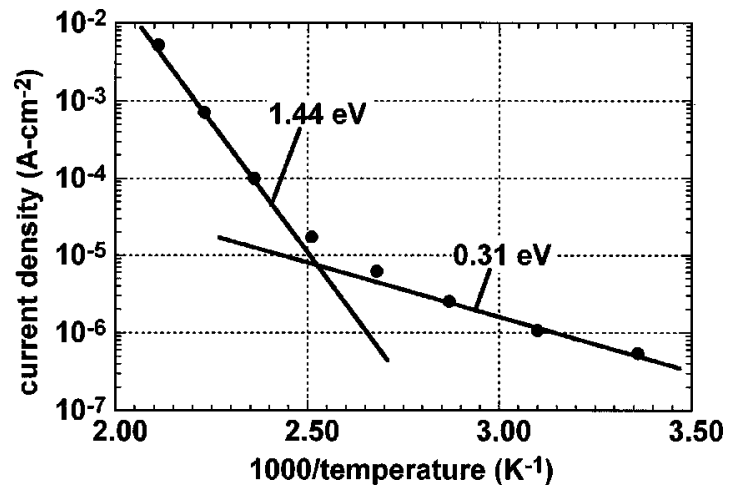

FIG. 4. Current vs $1 / T$ for a capacitor with a $40 \% \mathrm{Ta}_{2} \mathrm{O}_{5}-60 \% \mathrm{Al}_{2} \mathrm{O}_{3}$ alloy dielectric (Ref. 14). The energy of $0.31 \mathrm{eV}$ is for excitation into localized Ta $5 d$ trapping states, and the energy of $1.44 \mathrm{eV}$ is for thermal activation out of these Ta traps into Al s-like conduction band states. 
consistent with the MO model. The LMO calculations indicate a spatial localization of antibonding $d$ states on the TM atoms with a minimum energy approximately equal to the $n$ $d$ atomic state. The LMO model predicts that band gaps of the oxides, sulfides, and selenides for a given TM atom will scale with the anion atomic $p$-state energy as shown in Fig. 3(a). Band gaps of $\sim 3.0 \mathrm{eV}$ for $\mathrm{TiO}_{2}$, and $\sim 5.5-6.0 \mathrm{eV}$ for $\mathrm{ZrO}_{2}$ and $\mathrm{HfO}_{2}$ are consistent with the respective $d$-state energies of $\mathrm{Ti}, \mathrm{Zr}$, and $\mathrm{Hf},-11.05,-8.46$, and $-8.14 \mathrm{eV}^{13}$

The results of Fig. 3(b) are of particular importance for advanced Si devices. First, they show that the band offset energy for tunneling scales with atomic properties with obvious extensions to rare earth atom lanthanide oxides, $\mathrm{Gd}(\mathrm{Dy})_{2} \mathrm{O}_{3}$. They demonstrate that energies of localized $d$ states in elemental TM oxides scale with the energy difference between the Si conduction band and the TM atomic $d$ state, thereby providing an important atomic metric for high- $k$ gate dielectric evaluation and selection. The larger the energy separation between TM atom $n+1 s$ and $n d$ states, (i) the larger the conduction band offset energy between $\mathrm{Si}$ and a TM oxide, and therefore (ii) the lower the barrier for direct tunneling and/or interfacial and bulk electron trapping, each of which is important for device performance and reliability. However, there is an additional factor to consider in TM silicate and aluminate alloys. The AES data of Ref. 14 indicate systematic shifts in the energies of the Ta and $\mathrm{Al} p$ states as a function alloy composition. Recent synchrotron $\mathrm{X}$-ray photoelectron spectroscopy (SXPS) studies reveal these shifts as well. ${ }^{15}$ The data in Fig. 1 demonstrate that the lowest unoccupied $d$ states in TM metal silicate alloys are fixed in energy with respect to relatively deep core states. Therefore, alloy effects are also expected in the band offset energies. Based on the SXPS studies, ${ }^{15}$ these offset energies for TM $d$ states in silicate and aluminate alloys are expected to be a function of the chemical bonding environment of the alloys. For example, since the electronegativity difference between $\mathrm{Ta}$ and $\mathrm{Al}$ is small, $\sim 0.1$ on the Pauling scale, the shift is expected to be small, $\sim 0.1 \mathrm{eV}$, consistent with results in Fig. 4. However, electronegativity differences between $\mathrm{Zr}$ and $\mathrm{Si}$ are larger, $\sim 0.5-0.6$, and the energy of the $\mathrm{Zr}$ unoccupied $d$ states in $\mathrm{Zr}$ silicate alloys is anticipated to be several tenths of an $\mathrm{eV}$ smaller than the band offset between $\mathrm{Si}$ $\mathrm{ZrO}_{2}$.

Note added in proof: The calculations presented in Ref. 3 predict approximately equal conduction band offset energies with respect to $\mathrm{Si}$ for $\mathrm{Zr}(\mathrm{Hf}) \mathrm{O}_{2}$ and $\mathrm{Zr}(\mathrm{Hf}) \mathrm{SiO}_{4}$; however, XPS studies of our group, ${ }^{16}$ and Ref. 17 indicate that the $\mathrm{Zr}$ $3 d$ state binding energy is more than $0.5 \mathrm{eV}$ larger in $\mathrm{ZrSiO}_{4}$ than in $\mathrm{ZrO}_{2}$; i.e., it is more negative. This counter-intuitive difference is consistent with the principle of electronegativity equalization; i.e., the charge transfer out of $\mathrm{Zr}$ is larger in $\mathrm{ZrSiO}_{4}$ than in $\mathrm{ZrO}_{2}$ because the electronegativities of $\mathrm{Si}$ and $\mathrm{O}$ are each larger than that of $\mathrm{Zr}^{18}$ The results in Fig. 1 combined with the XPS results suggest that the lowest $\mathrm{Zr}$ antibonding $d$ state energy relative to the Si conduction band edge should decrease as the $\mathrm{Zr}$ content of a $\mathrm{Zr}$ silicate alloy is decreased resulting in increases in interfacial trapping and trap assisted tunneling. Calculations and XPS studies ${ }^{16}$ have demonstrated a quantitative difference between $\mathrm{Zr}$ silicate alloys and the $\mathrm{Ta}_{2} \mathrm{O}_{5}-\mathrm{Al}_{2} \mathrm{O}_{3} \cdot{ }^{13}$ Since the electronegativity difference between $\mathrm{Ta}$ and $\mathrm{Al}$ is smaller than the corresponding differences between $\mathrm{Si}$, and either $\mathrm{Zr}$ or $\mathrm{Hf}$, smaller changes in the XPS binding energies of Ta and Al with alloy composition are predicted and observed. ${ }^{13}$ This means that the conduction band offset energey of the Ta antibonding $d$ state should have a relatively small compositional dependence as has been discussed with respect to results in Fig. 4 . In contrast, the corresponding offset energy of a $\mathrm{Zr}(\mathrm{Hf})$ antibonding $d$ state in a $\mathrm{Zr}(\mathrm{Hf})$ silicate alloy should be reduced with respect to the offset energy between $\mathrm{Si}$ and $\mathrm{Zr}(\mathrm{Hf}) \mathrm{O}_{2}$ by a larger and compositionally dependent energy, anticipated to be approximately 0.3 to $0.5 \mathrm{eV}$ in the $\mathrm{SiO}_{2}$-rich silicate alloy regime.

This work was supported by the Office of Naval Research, the Air Force Office of Scientific Research, the Department of Energy and the SEMATECH/SRC Front End Processing Center. The NSLS is supported by the Department of Energy. We thank Shane Stadler for assistance with the XAS measurements.

${ }^{1}$ G. Wilk, R. W. Wallace, and J. M. Anthony, J. Appl. Phys. 89, 5243 (2001)

${ }^{2}$ S. Miyazaki (unpublished).

${ }^{3}$ J. Robertson and C. W. Chen, Appl. Phys. Lett. 74, 1168 (1999); J. Robertson, J. Vac. Sci. Technol. B 18, 1785 (2000).

${ }^{4}$ H. B. Gray, Electrons and Chemical Bonding (W. A. Benjamin, New York, 1962), Chap. 9; C. J. Ballhausen and H. B. Gray, Molecular Orbital Theory (W. A. Benjamin, New York, 1964), Chap. 8.

${ }^{5}$ P. A. Cox, Transition Metal Oxides (Oxford Science, Oxford, 1992), Chap. 2.

${ }^{6}$ G. Rayner, Jr., R. Therrien, and G. Lucovsky, Mater. Res. Soc. Symp. Proc. 611, c1 3.1 (2001).

${ }^{7}$ G. Lucovsky and G. Rayner, Jr., Appl. Phys. Lett. 77, 2912 (2000).

${ }^{8}$ L. A. Grunes, R. D. Leapman, C. D. Walker, R. Hoffman, and A. B. Kunz, Phys. Rev. B 25, 7157 (1982).

${ }^{9}$ Z. Y. Wu, F. Lemoigno, P. Gressier, G. Ouvard, P. Moreau, J. Rouxel, and C. R. Natoli, Phys. Rev. B 54, R11009 (1996).

${ }^{10}$ J. A. Wilson and A. D. Yoffe, Adv. Phys. 18, 193 (1969).

${ }^{11}$ J. A. Allen, G. Lucovsky, and J. C. Mikkelsen, Jr., Solid State Commun. 24, 367 (1977).

${ }^{12}$ W. A. Harrison, Elementary Electronic Structure (World Scientific, Singapore, 1999).

${ }^{13}$ R. S. Johnson, J. G. Hong, and G. Lucovsky, J. Vac. Sci. Technol. B 19, 1606 (2001).

${ }^{14}$ R. S. Johnson, J. G. Hong, and G. Lucovsky, J. Vac. Sci. Technol. A 19, 1353 (2001).

${ }^{15}$ M. Ulrich, R. S. Johnson, J. E. Rowe, and G. Lucovsky (unpublished).

${ }^{16}$ G. B. Rayner, Jr., K. Mai, M. Schultz, D. Kang, and G. Lucovsky (unpublished).

${ }^{17}$ M. J. Guittet, J. P. Crocombette, and M. Gautier-Soyer, Phys. Rev. B 63, 125117 (2001)

${ }^{18}$ R. T. Sanderson, Chemical Bonds and Bond Energy (Academic Press, New York, 1971), Chap. 2. 